\title{
The way forward
}

\section{Danielle Lemann}

Dr. med., Praxis für Hausarzt- und Komplementärmedizin, Mitglied FMH

1994 wurde das neue KVG von den Eidgenössischen Räten verabschiedet. Drei Krankenkassen und vier komplementärmedizinische Ärztevereinigungen unter der Leitung des «Forum für Freiheit im Gesundheitswesen FFG» machten für das Referendum mobil, so dass dieses in kurzer Zeit mit 148952 gültigen Unterschriften eingereicht werden konnte. Die Gegner des neuen KVG fanden in der Politik Gehör mit den Argumenten, das neue Gesetz sei zu dirigistisch und zu sehr der Schulmedizin verhaftet, zu teuer für die öffentliche Hand und die Prämienzahler. In der Abstimmung vom 4. Dezember 1994 wurde das neue KVG dann äusserst knapp angenommen.

Im Vorfeld gab Bundesrätin Dreifuss Versprechungen $\mathrm{ab}$, die Komplementärmedizin besser zu berücksichtigen. Sie hat diese Versprechen gehalten:

- Die fünf "wichtigsten» ärztlichen komplementärmedizinischen Disziplinen, d.h. Klassische Homöopathie, Traditionelle Chinesische Medizin, Anthroposophische Medizin, Phytotherapie und Neuraltherapie ${ }^{1}$ wurden von ihr als «umstritten» erklärt - mit der Auflage eines Nachweises von Wirksamkeit, Zweckmässigkeit und Wirtschaftlichkeit (WZW) gemäss Art. 32 KVG durch umfassende Studien im Rahmen eines mit 6 Mio. Franken grosszügig dotierten, auf 6 Jahre ausgelegten «Programms Evaluation Komplementärmedizin» (PEK).

Neuraltherapie betrachtet sich nicht mehr als der Komplementärmedizin zugehörig und trat 2011 aus der UNION aus.

2 Gründungsvorstand Dr. Ueli Heusser

(Gründungspräsident), Dr. Olivier Kappeler, Dr. Marcel Brander (erste Präsident), Dr. Andreas Beck, Dr. Brigitte Ausfeld, Dr. Valerio Rosinus, Dr. Klaus A. Halter, Dr. André Theurillat, Dr. Danielle Lemann (die beiden Ärztinnen kamen etwas später dazu, weil Frau Bundesrätin Dreifuss in allen Gremien eine Frauenquote erwartete, so auch in den Eidgenössischen Kommissionen EAK und ELK). medizin, forderte in der Folge 20 Arbeitsjahre und vier Gesundheitsminister.

Mit Schreiben vom 21. Februar 1996 hatte H.H. Brunner an M. Moser, Vizedirektor des damals noch für die Belange des KVG zuständigen Bundesamts für Sozialversicherungen, geschrieben:

«Die noch nicht einmal richtig begonnenen Diskussionen um die Pflichtleistungsbeurteilung komplementärmedizinischer Leistungen erfüllen mich mit grosser Sorge. Die komplementärmedizinischen Gesellschaften fordern immer klarer eine Einlösung der Zusagen, die nach ihrer Auffassung Frau Bundesrätin Dreifuss im Umfeld der KVG-Abstimmung gemacht hat. Namens der Verbindung der Schweizer Ärzte/FMH bitte ich Sie dringend darum, für eine Lösung der Problematik im Jahre 1996, dies mit Wirkung für das Jahr 1997, besorgt sein zu wollen ...»

Das Interesse der Fachwelt war gross, auch die Öffentlichkeit, vertreten durch 36 Journalisten, wartete gespannt auf die Studienergebnisse.

Auf den 21. April 2005 hatte die UNION die entscheidende wissenschaftliche Fachtagung zur Präsentation und Diskussion der PEK-Resultate im randvoll besetzten Auditorium Maximum der Universität Bern angesetzt. Das Interesse der Fachwelt war gross, auch die Öffentlichkeit, vertreten durch 36 Journalisten, wartete gespannt auf die Studienergebnisse.

Aber seit der Amtsübernahme des neuen Gesundheitsministers, Pascal Couchepin, und nachdem klar wurde, dass die ärztliche Komplementärmedizin tendenziell kostengünstiger ist, hatte der Wind gedreht. Die 17 Referenten wurden mit Drohbriefen unter Druck gesetzt, 14 Referenten zogen ihre Beiträge zurück - damit blieben fast alle geplanten wissenschaftlichen Publikationen zum PEK bis heute unterdrückt. Couchepin hatte ein ausgesprochen ambivalentes Verhältnis zur Komplementärmedizin, was ihn zuweilen zu irritierenden Statements veranlasste.

Die Anträge der fünf Fachgesellschaften zur definitiven Aufnahme in der OKP wurden durch die Eidgenössische Leistungs- und Grundsatzkommission (ELGK) 2005 abgelehnt mit der hauptsächlichen Begründung, dass "das Gebot der Wirksamkeit» nicht erfüllt sei. Fünf Jahre später erfolgte die erneute Zurückweisung 
der wesentlich nachgebesserten Anträge, diesmal jedoch, weil die Wirtschaftlichkeit nicht hinreichend belegt sei, während hinsichtlich Wirksamkeit jetzt von einer «Gleichwertigkeit mit konventioneller (schulmedizinischer) Behandlung» gesprochen wurde. Die Kostengünstigkeit wurde in der Folge mit verschiedenen Publikationen gut belegt.

Die UNION hielt selber in allen Auseinandersetzungen an der Wissenschaftlichkeit fest, führte dazu nebst den regelmässigen Fachtagungen der Mitgliederorganisationen auch fachübergreifende SGAM-anerkannte Kongresse durch. Bereits 2002 fand eine Tagung zum Problem zunehmender Antibiotikaresistenzen statt, einem Thema, das zwölf Jahre später vom Bund im Projekt «Nationale Strategie Antibiotikaresistenzen (STAR)» aufgegriffen wurde. Speziell bei der antibiotikafreien Behandlung von Atemwegsinfektionen haben komplementärmedizinisch geschulte Ärzte jahrzehntelange Erfahrung.

Im Gefolge der Abstimmung vom 19. Mai 2009, in der eine deutliche $2 / 3$-Mehrheit der Schweizer Stimmberechtigten «die Berücksichtigung der Komplementärmedizin» forderten, kam es zu einer nun sachgemässen Bearbeitung der Frage des adäquaten WZW-Nachweises unter umsichtiger Führung durch das Departement des Innern, mittlerweile unter BR Didier Burkhalter. Seit 2011 arbeitet eine «Begleitgruppe» des EDI konsequent an der Umsetzung der Kernforderungen der Volksinitiative. Darunter fällt die definitive Aufnahme der ärztlichen Komplementärmedizin in der OKP ebenso wie die Berücksichtigung in den Lern- und Prüfungskatalogen der Medizinalberufe, die Förderung der Forschung und die Sicherung der bewährten Heilmittelvielfalt. Hier besteht eine intensive Zusammenarbeit der UNION mit der breit abgestützten politischen Dachorganisation Dakomed.

Am 5. Jahrestag der Volksabstimmung präsentierte der neue Gesundheitsminister Alain Berset am 30. April 2014 die entscheidende "Road Map», die den 20-jährigen Anerkennungsprozess der ärztlichen Komplementärmedizin mittels entsprechender Verordnungsänderungen, aber unter Sicherung der Qualität, nun zu einem Abschluss führen soll. Eine 20-köpfige Expertengruppe unter Leitung des BAG mit Vertretern der Universitäten, von BAG, FMH, SAMW, Konsumentenschutz SKS, santésuisse und curafutura erarbeitete in der Folge dazu die wissenschaftlichen Grundlagen. Dabei wurde anerkannt, dass in der Schweiz bereits durch die hohen Anforderungen an die Ausbildung im Rahmen der FMH-anerkannten Fähigkeitsausweise die Latte hoch angesetzt ist, ferner, dass auch in der ärztlichen Komplementärmedizin mittlerweile eine Fülle von klinischen Studien, durchaus auch von hohem Evidenzlevel, vorliegt. Besonders in der Behandlung von Kindern, Schwangeren und alten Menschen und bei chronischen Krankheiten hat die Komplementärmedizin in der Praxis längst Bedeutung erlangt, speziell auch in präventivmedizinischer Hinsicht. Wissenschaftlich findet sich dieser Prozess gut wiedergegeben in einem Beitrag im European Journal of Integrative Medicine unter dem Titel «Evidence-informed integrative systems», richtungsweisend ergänzt mit dem Untertitel: «The way forward» [1].

\section{Das Hauptanliegen der UNION, die definitive} Anerkennung der ärztlichen Komplementärmedizin, forderte in der Folge 20 Arbeitsjahre und vier Gesundheitsminister.

Unterdessen hat die UNION erkannt, dass die ärztliche Komplementärmedizin eine Rückbesinnung auf ihr Selbstverständnis und ihren Auftritt vornehmen muss. Oft ist für die «Basis» nicht ersichtlich, wozu es diese Dachorganisation braucht. Den 20-jährigen Weg der UNION fasste der eben zurückgetretene Präsident des Schweizer Vereins Homöopathischer Ärztinnen und Ärzte (SVHA) so zusammen:

«Von einer verordneten Zweckstruktur zur Trägerin einer komplementärmedizinischen Kultur.» Die UNION bemüht sich weiterhin, diesem Anspruch gerecht zu werden.

\footnotetext{
Literatur

1 Sundberg T et al. Evidence-informed integrative care systems - The way forward. Eur J Integr Med 2013, http://dx.doi. org/10.1016/j.eujim.2013.09.005
} 